\title{
Effects of intrinsic stochasticity on delayed reaction-diffusion patterning systems
}

\author{
Thomas E. Woolley, ${ }^{1,2, *}$ Ruth E. Baker, ${ }^{1}$ Eamonn A. Gaffney, ${ }^{1}$ Philip K. Maini, ${ }^{1,3}$ and Sungrim Seirin-Lee ${ }^{4}$ \\ ${ }^{1}$ Centre for Mathematical Biology, Mathematical Institute, University of Oxford, 24-29 St Giles', Oxford, OX1 3LB, United Kingdom \\ ${ }^{2}$ Oxford Centre for Collaborative Applied Mathematics, Mathematical Institute, University of Oxford, 24-29 St Giles', \\ Oxford, OX1 3LB, United Kingdom \\ ${ }^{3}$ Oxford Centre for Integrative Systems Biology, Department of Biochemistry, University of Oxford, South Parks Road, \\ Oxford, OX1 3QU, United Kingdom \\ ${ }^{4}$ Center for Developmental Biology, RIKEN, Minatojima-Minamimachi 2-2-3, Kobe 650-0047, Japan
}

(Received 26 February 2012; published 22 May 2012)

\begin{abstract}
Cellular gene expression is a complex process involving many steps, including the transcription of DNA and translation of mRNA; hence the synthesis of proteins requires a considerable amount of time, from ten minutes to several hours. Since diffusion-driven instability has been observed to be sensitive to perturbations in kinetic delays, the application of Turing patterning mechanisms to the problem of producing spatially heterogeneous differential gene expression has been questioned. In deterministic systems a small delay in the reactions can cause a large increase in the time it takes a system to pattern. Recently, it has been observed that in undelayed systems intrinsic stochasticity can cause pattern initiation to occur earlier than in the analogous deterministic simulations. Here we are interested in adding both stochasticity and delays to Turing systems in order to assess whether stochasticity can reduce the patterning time scale in delayed Turing systems. As analytical insights to this problem are difficult to attain and often limited in their use, we focus on stochastically simulating delayed systems. We consider four different Turing systems and two different forms of delay. Our results are mixed and lead to the conclusion that, although the sensitivity to delays in the Turing mechanism is not completely removed by the addition of intrinsic noise, the effects of the delays are clearly ameliorated in certain specific cases.
\end{abstract}

DOI: 10.1103/PhysRevE.85.051914

PACS number(s): 87.18.Hf, 87.18.Tt, 82.20.Uv

\section{INTRODUCTION}

One of the fundamental unanswered questions of developmental biology remains that of how spatiotemporal complexity arises. Using relatively few homologous and evolutionarily conserved pathways the biological world produces multiple diverse morphological patterns [1]. Primarily, this selforganization is thought to arise through seemingly identical cells undergoing differential gene expression [2]. These differentiated cells are then able to express different proteins and thus create, and respond to, heterogeneous environments. Of course, there will always be a certain degree of variability between individual living systems. Even asexual reproduction can result in different phenotypes from the same genotype if individuals are subject to differing environmental conditions [3]. However, although numerous sources of intrinsic and extrinsic noise exist, biological processes are able to consistently exhibit similar results, e.g., the same number of digits in chick wings and legs [4,5]. Consequently, the development of patterns through gene expression requires rigorous spatiotemporal coordination, which can be exerted by both long-range signals, such as diffusible-morphogen gradients [6], and short-range signals, as exemplified by cell-to-cell communication via signaling pathways [7]. Critically, development can proceed at an extremely rapid rate, emphasizing the likely presence of temporal coordination and regulation. For example, the basic body plan of the zebrafish is established in under $24 \mathrm{~h}[8]$.

*woolley@maths.ox.ac.uk
In this paper we focus on the particular mechanism of Turing's diffusion-driven instability [9]. Diffusion is well known for its homogenizing properties. However, Turing postulated that, when coupled with certain reactions, diffusion could lead to a persistent inhomogeneous state, created through a spontaneous symmetry breaking of initial near-homogeneous populations. There is certainly evidence to support the presence of gene expression for prospective Turing morphogens, e.g. Nodal and Lefty in zebrafish, with in situ hybridisation and mRNA expression [10-14].

However, many applications of Turing's mechanism assume instantaneous biochemical reactions for regulated protein production $[15,16]$. This can quickly be seen to be inadequate as the process of producing a gene product is a long and complicated one.

First, a linear polymeric ribonucleic acid (RNA) molecule is produced in the cell nucleus. This RNA molecule is an exact copy of the relevant gene sequence and is modified into a form called messenger RNA (mRNA). The mRNA is then transported into the nuclear membrane, where it is used as a blueprint for protein synthesis. In particular, the process of mRNA translation involves the polymerization of thousands to millions of amino acids [17]. Given the complexity of this mechanism it should not be surprising that a delay occurs between the initiation of protein translation and the point at which mature proteins are observed. The exact delay depends both on the length of the sequence being read and the sequence being created. However, typically the delay ranges from tens of minutes to as long as several hours [18,19]. Additionally, since inter- and intracellular reactions frequently operate in noisy conditions [20-22], we are interested in comparing the results of delayed deterministic 
simulations with the, perhaps, more appropriate stochastic formalism.

For Turing patterns to exist in a two species reaction system it is well known that diagonal elements of the Jacobian must be of opposite signs [23], namely the Jacobian must have one of the following forms (including the symmetries $+\leftrightarrow-$ ):

$$
J_{c}=\left(\begin{array}{c}
++ \\
--
\end{array}\right), \quad J_{p}=\left(\begin{array}{c}
+- \\
+-
\end{array}\right) .
$$

A kinetic mechanism for which the Jacobian is of type $J_{c}$ (type $J_{p}$ ) is said to be a cross (pure) activator-inhibitor mechanism [24].

In physical terms pure kinetics $\left(J_{p}\right)$ comprise a short-range self-activator which is inhibited by a long-range self-inhibitor; hence the activator and inhibitor are in phase. The cross kinetic form $\left(J_{c}\right)$ causes a self-activator to deplete a self-inhibitor. This, in turn, feeds production of the activator, causing the spatial oscillations to be in antiphase. The distinction between pure and cross kinetics is rarely made as, in general, they behave similarly [23]. One notable difference that can be observed in the case of Turing patterns on a growing domain is that the activator population in a pure kinetic system favors a peak doubling transition between patterning states, whereas the activator in a cross kinetic system tends to peak split [25]. However, in either case, once a pattern transition has occurred as a result of domain growth we are left with a pattern that contains double the number of initial concentration peaks. Thus, unless the mechanism by which the pattern changes can be experimentally observed or the morphogens can be shown to be in or out of phase, we are left without an exact way of distinguishing between the two types of kinetics from observations at the macroscale.

In contrast, when delays are added to pure and cross kinetics systems large differences can be observed in their behavior [26,27], at least for Gierer-Meinhardt kinetics [28] and Schnakenberg kinetics [29] (originally proposed by Gierer and Meinhardt [28]), which are exemplars of pure and cross kinetics, respectively. In particular, Gierer-Meinhardt kinetics contain a negative feedback loop which can work in conjunction with the time delay to produce oscillations, which are not seen with Schnakenberg kinetics [27]. Here we extend our studies, not only considering Gierer-Meinhardt and Schnakenberg kinetics but including delays in the glycolysis kinetic system [30,31] and an adapted version of the cubic autocatalytic kinetic system [32], which are further examples of cross and pure kinetics, respectively.

A further complication in modeling delayed systems is due to the observation that the specification of where and how the delay occurs may not be unique. Here, two extreme, biologically motivated cases are considered. The dominant view is that signal transduction occurs via reversible-ligand binding. This assumes that the signal for the production of morphogens is produced through reversible morphogen binding on the cell surface with an associated gene expression delay between the initiation signal and protein production $[33,34]$. Once the cell begins to form morphogen products, particles bound to the surface of the cell are able to return to the bulk and initiate further reactions. An alternative signaling mechanism, known as ligand internalization, has increasing empirical evidence for its existence. In this case the signal is transduced via ligands that are internalized [35,36]. Thus, instead of the particles being able to leave the binding sites of the external cell membrane once production has been initiated, the binding particles are removed from the system.

Our interest in stochastically simulating delayed equations stems from recent work in which we questioned how the Turing mechanism was affected by factors such as stochasticity [37]. It was seen that intrinsic stochasticity can actually enhance production of the patterned state as, not only do stochastic Turing structures form outside of the deterministic parameter domain, the noisy simulations are able to produce the patterned state quicker than their deterministic analogues. Thus our intention is to use numerical simulations to investigate whether intrinsic noise can overcome the sensitivity of Turing's mechanism to delays, especially the extreme patterning lags observed in delayed systems.

In the case of delayed partial differential equations (PDEs) analytic results concerning linear stability are difficult to derive as the eigenvalue equation is transcendental and timedependent. Furthermore, it has been shown that standard linear analysis is typically unable to capture the dynamics of the full nonlinear system [26]. Thus full linear analysis of the delayed system is not considered here. Instead, we focus on numerically comparing the stochastic and deterministic systems, in order to observe the effects of stochasticity on delayed Turing systems.

Initially, in Sec. II, we consider the effect of altering the initial conditions on the pattern formation time scale for undelayed systems and use this to justify our interest in adding stochastic effects to delayed systems. After observing how intrinsic noise can reduce the patterning lag we progress to adding a representation of gene expression delay into morphogen-regulated-morphogen-production of the Turing kinetics in Sec. III and discuss how these are to be simulated, deterministically and stochastically in Sec. IV. The results of simulating delayed pure and cross Turing kinetics are illustrated in Sec. V. Finally, in Sec. VI we bring together our results to assess the biological applicability of Turing patterns in delayed systems.

\section{SENSITIVITY TO INITIAL CONDITIONS}

One factor that can alter the time taken between system initiation and pattern realization is the form of initial condition. In this section we use the Schnakenberg kinetics to demonstrate how noise can remove this sensitivity. Although originally derived in 1972 [28], the Schnakenberg kinetics were popularized in 1979, where they were derived as one of eleven sets of kinetics that demonstrated the necessary properties for limit cycle behavior, these being that the system needs at least three reactions with at least one of those being autocatalytic [29]. Since then extensive analysis has been performed on the Schnakenberg kinetics [38-40], which can be thought of as a simplified version of the Brusselator model [41]. For populations $U$ and $V$, the Schnakenberg reactions can be written as

$$
\emptyset \underset{\left[c_{-1}\right]}{\stackrel{c_{1}}{\rightleftharpoons}} U, \quad \emptyset \stackrel{c_{2}}{\rightarrow} V, \quad 2 U+V \stackrel{c_{3}}{\rightarrow} 3 U
$$


where the $c_{n}$ are deterministic reaction rates and $\emptyset$ represents substances which are not considered. By letting $\phi$ and $\psi$ be the concentrations of $U$ and $V$, respectively, and allowing the reactants to diffuse, the PDE system becomes

$$
\begin{gathered}
\frac{\partial \phi}{\partial t}=D_{\phi} \frac{\partial^{2} \phi}{\partial x^{2}}+c_{1}-c_{-1} \phi+c_{3} \phi^{2} \psi, \\
\frac{\partial \psi}{\partial t}=D_{\psi} \frac{\partial^{2} \psi}{\partial x^{2}}+c_{2}-c_{3} \phi^{2} \psi,
\end{gathered}
$$

where throughout this paper the equations are simulated on finite one-dimensional domains with homogeneous Neumann boundary conditions prescribed at each end. Alongside these deterministic equations we also stochastically simulate the reactions in Eq. (2) using the software Dizzy 1.11.4 [42] to implement a standard Gillespie stochastic simulation algorithm (SSA) $[43,44]$. The methods of simulation are discussed further in Sec. IV and Appendix B. Note that the stochastic system uses stochastic reaction rates, $\kappa_{n}$, which have been scaled such that the theoretical homogeneous steady state of the stochastic population is $\Omega$ times larger than the deterministic concentration at the homogeneous steady state. Thus $\Omega$ is a population scale that links the discrete, stochastic description of the microscopic scale to the continuous, deterministic macroscopic scale [37,45-49] and, thus, approximately, $\phi \Omega \approx U$ and $\psi \Omega \approx V$. Here, unless otherwise stated, $\Omega=100$.

In Figs. 1(a)-1(c) the same deterministic system is simulated three times with three different initial conditions. In each case, a normally distributed random perturbation with mean zero and standard deviation $\sigma$ was added to each spatial point of the homogeneous steady state. If, upon the addition of this random number, the initial condition was negative, the absolute value of the number was taken. Figures 1 (a)-1(c) show the effect of decreasing the standard deviation over the three simulations. Clearly, we can see that the size of perturbation affects the resulting time between pattern initiation and pattern formation.

Since the normal distribution produces white noise all spatial frequencies have the same power. We also tested uniformly distributed random initial conditions and pseudorandom initial conditions in order to see if these affected the pattern initiation time in any particular way (results not shown). Although these different initial conditions may excite all wave modes, they will not all have the same power; thus certain frequencies may dominate. However, it is observed that altering the initial condition in this manner has little effect on the time taken to form patterns, while altering the amplitude of the noise produces similar results to those seen in Fig. 1.

The dependence of the time taken to pattern on the initial condition is easy to understand; if the deterministic system is initialized at exactly the homogeneous steady state then (ignoring computational error) the system will remain at the steady state. However, any perturbation, no matter how small, will destabilize this state. Initially, the small perturbation grows approximately exponentially. By decreasing $\sigma$ we decrease the variance of the noise about the homogeneous steady state and, hence, the initial amplitude of this perturbation. Thus, although the wave mode amplitude increases exponentially, it has to increase from a state that is very close to homogeneity

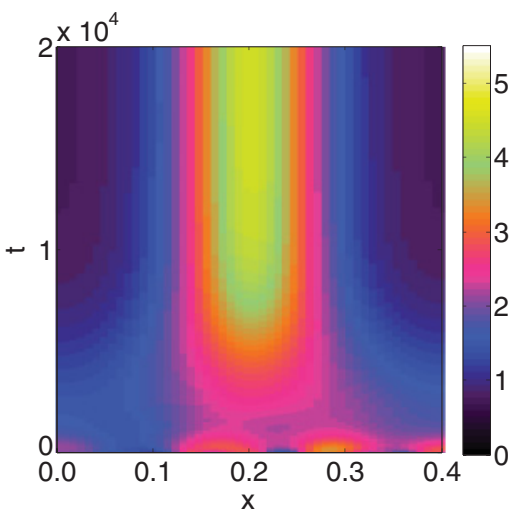

(a) $\sigma=1$

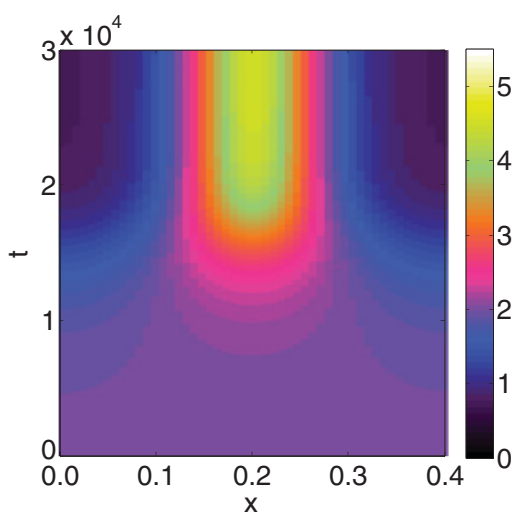

(c) $\sigma=1 / 100$

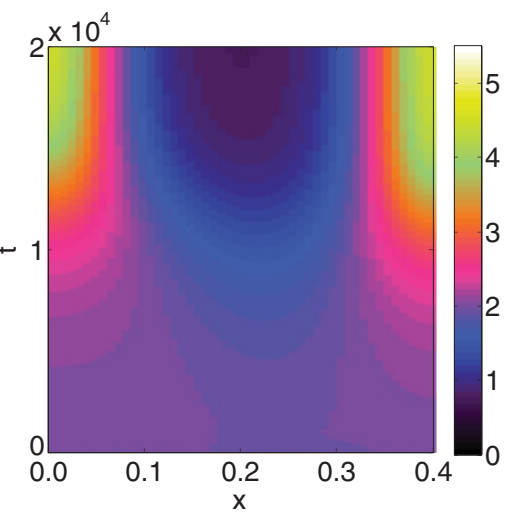

(b) $\sigma=1 / 10$

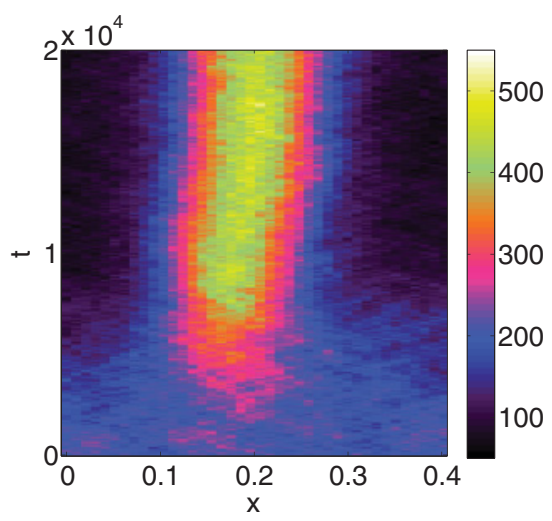

(d)
FIG. 1. (Color online) (a)-(c) Deterministic pattern formation from homogeneous steady-state initial conditions with normally distributed noise (mean zero and standard deviation, $\sigma$, given under each figure) added to the deterministic initial conditions. (d) Stochastic pattern formation from homogeneous steady-state initial conditions. The illustrated system is the Schnakenberg kinetics, Eqs. (3) and (4). The deterministic system was simulated using an explicit Euler stepping method and the stochastic system was simulated using a standard Gillespie algorithm. Parameters are $D_{u}=10^{-6} \mathrm{~mm}^{2} / \mathrm{s}, D_{v}=10^{-4} \mathrm{~mm}^{2} / \mathrm{s}, c_{1}=$ $10^{-3} \mathrm{~mol} /(\mathrm{mm} \mathrm{s}), c_{-1}=2 \times 10^{-3} \mathrm{~s}, c_{2}=3 \times$ $10^{-3} \mathrm{~mol} /(\mathrm{mm} \mathrm{s})$, and $c_{3}=10^{-3} \mathrm{~mm}^{2} /\left(\mathrm{mol}^{2}\right.$ $\mathrm{s})$. The boundary conditions are zero flux, the temporal axis is in seconds, and the spatial axis is in millimeters. The color axis scale is in moles $/ \mathrm{mm}$ for the deterministic simulations and particles/mm for the stochastic simulations. The boundary conditions and axis scales are the same for all the following figures, unless otherwise stated. 


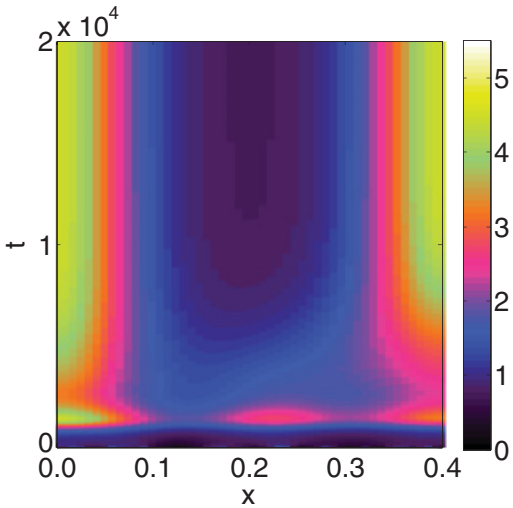

(a) $\sigma=1$

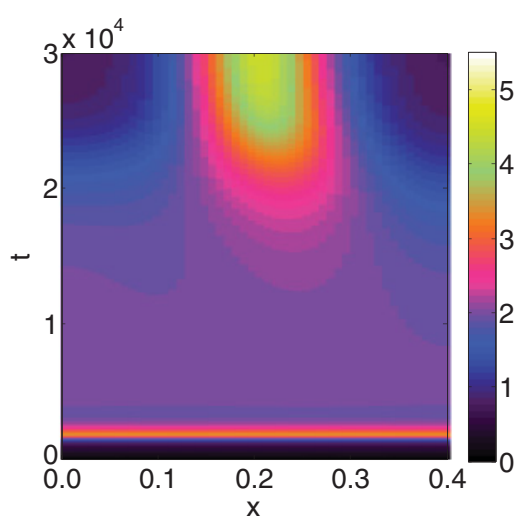

(c) $\sigma=1 / 100$

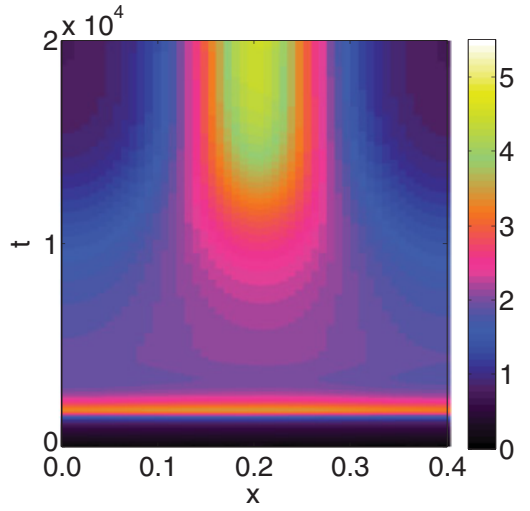

(b) $\sigma=1 / 10$

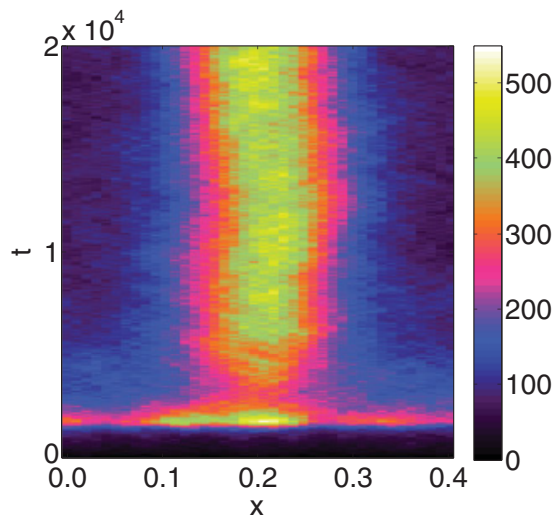

(d) when $\sigma=1 / 100$ and so the time taken for patterns to appear is increased. It is worth noting that, since the wave modes grow exponentially, we expect a logarithmic relationship between the size of the perturbation and the increase in time.

The deterministic simulations, Figs. 1(a)-1(c), can then be compared with an analogous stochastic simulation in Fig. 1(d). Here, the initial condition was the uniform steady state exactly and it is the intrinsic noise which destabilizes the homogeneous state. By considering Fig. 1 we observe that the patterning time scale of the stochastic simulation is most similar to the deterministic simulation which had the initial condition with largest standard deviation. Critically, it is seen that by adding noise to the initial condition of the stochastic system the time scale of pattern formation is not affected (results not shown). Thus, not only does the inclusion of stochasticity reduce the sensitivity of the system to initial conditions, it also renders patterns much more quickly than the deterministic system, particularly when the initial condition is very close to the homogeneous steady state. This is an important observation since the initial stochasticity added to initialize many deterministic simulations is generated on an ad hoc basis. Here, we see evidence to suggest that characterizing the initial conditions correctly as well as incorporating intrinsic noise can have dramatic effects on the patterning time scale, when compared to that of the deterministic simulations.

Another assumption that we need to address is that all simulations produced thus far have been initiated from the homogeneous steady state. We should question whether these initial conditions are realistic in a biological sense. Certainly, in vitro morphogens could be added at the beginning of an experiment to reproduce steady-state conditions [50]. However, in vivo it will take time for morphogen concentrations to build up to sufficient levels before patterning can occur [4]. In Fig. 2 we simulate the exact same system as in Fig. 1, except that the initial conditions are set to zero in the stochastic case and zero with normally distributed noise in the deterministic case. Immediately, we see that the standard deviation has much more influence on the patterning time scale than the change in the initial condition. We should also note that the deterministic formulation of the system is actually invalid when we start sufficiently close to the zero state as we are violating the assumption that there are enough reactant particles to treat the morphogens as continuous quantities, rather than discrete particles.

Now that we have illustrated that intrinsic noise has the ability to remove the sensitivity of the diffusion-driven instability to initial conditions and increase the speed of the patterning mechanism, we apply the stochastic formalism to the case of delayed reaction kinetics. In the next section we discuss how kinetic delays are added to the deterministic and stochastic formulations.

\section{MATHEMATICALLY MODELING DELAYED KINETICS}

There are multiple ways of including delays in mathematical systems and current biological understanding is often insufficient to distinguish between them. Thus we consider two biologically motivated options, reversible-ligand binding and ligand internalization, and explore the following four 
model systems: Schnakenberg, glycolysis, Gierer-Meinhardt, and cubic autocatalysis [28,30-32]. The Schnakenberg case is considered in full detail with the other three cases being similar. Their respective kinetic forms are given in Appendix A.

\section{A. Schnakenberg system}

First, we consider the Schnakenberg kinetics with reversible-ligand binding. We place the delay in the nonlinear production term, as morphogen-regulatedmorphogen-production induces gene expression delays in protein production. The reactions,

$$
\emptyset \stackrel{\kappa_{1}}{\rightarrow} U, \quad U \stackrel{\kappa_{-1}}{\rightarrow} \emptyset, \quad \emptyset \stackrel{\kappa_{2}}{\rightarrow} V,
$$

are kept the same as in Sec. II. The delayed reaction,

$$
2 U+V \stackrel{\kappa_{3}, \text { delay } \tau}{\longrightarrow} 3 U,
$$

is encoded as the two-stage process. For the reversible-ligand binding representation of morphogen production we have

$$
2 U+V \stackrel{\kappa_{3}}{\rightarrow} 2 U+W, \quad W \stackrel{\text { delay } \tau}{\longrightarrow} U .
$$

Using the Law of Mass Action and relating the stochastic reaction rates, $k_{n}$, to their deterministic analogues, $c_{n}$, through an appropriate scaling of $\Omega$ [47] we derive the following set of PDEs:

$$
\begin{gathered}
\frac{\partial \phi}{\partial t}=D_{\phi} \frac{\partial^{2} \phi}{\partial x^{2}}+c_{1}-c_{-1} \phi \\
+c_{3} \underbrace{\phi(x, t-\tau)^{2} \psi(x, t-\tau)}_{\text {Gain one particle of } U \text { from delayed reaction at time } t-\tau .} \\
\frac{\partial \psi}{\partial t}=D_{\psi} \frac{\partial^{2} \psi}{\partial x^{2}}+c_{2}-c_{3} \underbrace{\phi^{2} \psi}_{\text {Lose one particle of } V \text { from reaction at time } t .}
\end{gathered}
$$

where only the delayed arguments have been shown. All of the other $\phi$ and $\psi$ functions are evaluated at $(x, t)$. It should be noted that the production of proteins occurs at the same spatial point as their initiation, for, although the proteins are able to diffuse, the cells which produce them cannot move.

In order to include the ligand internalization delay in the Schnakenberg system we keep reactions (5) the same as before and, once again, apply the delay to the cubic nonlinearity,

$$
2 U+V \stackrel{\kappa_{3}}{\rightarrow} W, \quad W \stackrel{\text { delay } \tau}{\longrightarrow} 3 U
$$

which models total internalization the $U$ and $V$. Explicitly, at the present time two particles of $U$ and one particle of $V$ are internalized and, thus, removed from the active populations. However, we also gain three particles of $U$ from the delayed reactions at a time $\tau$ in the past; thus the ligand internalized form is

$$
\begin{aligned}
\frac{\partial \phi}{\partial t}= & D_{\phi} \frac{\partial^{2} \phi}{\partial x^{2}}+c_{1}-c_{-1} \phi+c_{3}[\underbrace{3 \phi(x, t-\tau)^{2} \psi(x, t-\tau)}_{\begin{array}{c}
\text { Gain three particles of } U \\
\text { from delayed reaction. }
\end{array}} \\
& -\underbrace{2 \phi^{2} \psi}_{\begin{array}{c}
\text { Lose two particles of } U \\
\text { from present reaction. }
\end{array}}], \\
\frac{\partial \psi}{\partial t}= & D_{\psi} \frac{\partial^{2} \psi}{\partial x^{2}}+c_{2}-c_{3} \underbrace{\phi^{2} \psi}_{\text {Lose one particle of } V \text { from present reaction. }}
\end{aligned}
$$

\section{B. Glycolysis system}

The second form of cross kinetics we consider are known as the glycolysis kinetics [30,31]. The reversible-ligand binding PDE formulation is

$$
\begin{gathered}
\frac{\partial \phi}{\partial t}=D_{\phi} \frac{\partial^{2} \phi}{\partial x^{2}}+c_{1}-c_{2} \phi-c_{3} \phi \psi^{2}-c_{6} \phi \psi^{2}, \\
\frac{\partial \psi}{\partial t}=D_{\psi} \frac{\partial^{2} \psi}{\partial x^{2}}+c_{4} \phi-c_{5} \psi+c_{6} \phi(t-\tau) \psi(t-\tau)^{2},
\end{gathered}
$$

while the ligand internalization PDE formulation is

$$
\begin{aligned}
\frac{\partial \phi}{\partial t}=D_{\phi} \frac{\partial^{2} \phi}{\partial x^{2}}+c_{1}-c_{2} \phi-c_{3} \phi \psi^{2}-c_{6} \phi \psi^{2} \\
\frac{\partial \psi}{\partial t}=D_{\psi} \frac{\partial^{2} \psi}{\partial x^{2}}+c_{4} \phi-c_{5} \psi-c_{6} 2 \phi(t-\tau) \psi(t-\tau)^{2} \\
+3 c_{6} \phi(t-\tau) \psi(t-\tau)^{2} .
\end{aligned}
$$

\section{Gierer-Meinhardt system}

As discussed in Sec. I, the effects of the delay on particular types of pure and cross kinetics have been seen to be very different. Thus, in this section, we continue our investigation into the effects of delays on stochastic equations by considering the case of the Gierer-Meinhardt kinetics. The delay is, once again, added through the nonlinear production terms, but this time there are two such reactions. Although we could retard each delayed reaction separately with a different time scale, our focus is primarily on highlighting the general influences of noise in both deterministic and stochastic systems. Hence the delay in both equations is the same. Note that in Eq. (A5) we have adapted the propensity function of the standard GiererMeinhardt autocatalytic term, $\kappa_{3} / V$, to $\kappa_{3} /(V+0.001)$. This is to remove the problematic singularity that may occur if the $V$ population decreases to zero, as can occur in deterministic delayed systems [27]. This reaction system gives rise to a ligand-reversible binding system of the form

$$
\begin{gathered}
\frac{\partial \phi}{\partial t}=D_{\phi} \frac{\partial^{2} \phi}{\partial x^{2}}+c_{3} \frac{\phi(x, t-\tau)^{2}}{\psi(x, t-\tau)+10^{-5}}+c_{1}-c_{2} \phi, \\
\frac{\partial \psi}{\partial t}=D_{\psi} \frac{\partial^{2} \psi}{\partial x^{2}}+c_{4} \phi(x, t-\tau)^{2}-c_{5} \psi .
\end{gathered}
$$

The different constants in the nonlinear term, i.e., $\kappa_{3} /(V+$ $0.001)$ in the stochastic propensity function, and $c_{3} /(\psi+$ $10^{-5}$ ) in the deterministic propensity function, are needed to correct for the population scale, $\Omega=100$, which relates the stochastic population and deterministic concentration, as noted 
in Sec. II. The ligand-internalization PDE formulation is

$$
\begin{gathered}
\frac{\partial \phi}{\partial t}=D_{\phi} \frac{\partial^{2} \phi}{\partial x^{2}}+c_{3}\left(3 \frac{\phi(x, t-\tau)^{2}}{\psi(x, t-\tau)+10^{-5}}-2 \frac{\phi^{2}}{10^{-5}+\psi}\right) \\
+2 c_{4}\left[\phi(x, t-\tau)^{2}-\phi^{2}\right]+c_{1}-c_{2} U \\
\frac{\partial \psi}{\partial t}=D_{\psi} \frac{\partial^{2} \psi}{\partial x^{2}}+c_{4} \phi(x, t-\tau)^{2}-c_{5} \psi
\end{gathered}
$$

\section{Cubic autocatalytic system}

The second form of pure kinetics we consider are known as the cubic autocatalytic kinetics [32]. Originally, these were derived to make the origin the homogeneous steady state; hence the populations could become negative. In order to remove this problem the reactions which affect $U$ have been made to depend on having at least one particle of $U$ present. For example, originally the $U$ population was removed at a rate proportional to $\kappa_{1}$. This has been altered to make the rate proportional to $\kappa_{1} U$. The reversible-ligand binding form is

$$
\begin{gathered}
\frac{\partial \phi}{\partial t}=D_{\phi} \frac{\partial^{2} \phi}{\partial x^{2}}-c_{1} \phi-c_{2} \phi^{2}+c_{3} \phi(t-\tau) \psi(t-\tau) \\
\frac{\partial \psi}{\partial t}=D_{\psi} \frac{\partial^{2} \psi}{\partial x^{2}}+c_{4}-c_{5} \phi-c_{6} \psi+c_{7} \psi(t-\tau)^{2}-c_{8} \psi^{3},
\end{gathered}
$$

while the ligand internalization form of the delayed reactions is

$$
\frac{\partial \phi}{\partial t}=D_{\phi} \frac{\partial^{2} \phi}{\partial x^{2}}-c_{1} \phi-c_{2} \phi^{2}+2 c_{3} \phi(t-\tau) \psi(t-\tau)-c_{3} \phi \psi,
$$

$$
\begin{aligned}
\frac{\partial \psi}{\partial t}= & D_{\psi} \frac{\partial^{2} \psi}{\partial x^{2}}+c_{4}-c_{5} \phi-c_{6} \psi+c_{3} \phi(t-\tau) \psi(t-\tau) \\
& -c_{3} \phi \psi+3 c_{7} \psi(t-\tau)^{2}-2 c_{7} \psi^{2}-c_{8} \psi^{3}
\end{aligned}
$$

\section{NUMERICAL METHODS}

Since we are dealing with delayed processes our computational scheme must allow us to keep track of the history of the simulated variables in order to allow past populations to affect the present state. Although there are a number of ways of achieving this deterministically, which can be extended to encapsulate stochastic effects [51], we choose to use a "time bin" method. The delay time, $\tau$, is discretized into $M \in \mathbb{N}$ bins of size $\tau / M$. This is implemented as it is too computationally costly to keep track of every single time step [51], particularly in the case of the stochastic simulations where each reaction changes the state of a very small number of particles. In the deterministic case we use a time-explicit Euler stepping algorithm [27,52], which is discussed further in Appendix B.

The stochastic simulation of the delay needs further elucidation. In contrast to a deterministic delay, where we simply keep track of the population history and then update the present population based on this history, we create a new delayed population, $W$, and split a single delayed reaction up into two distinct actions; the creation of the delayed population and its decay to the products, as discussed in Sec. III and Appendix A. We then use the software Dizzy 1.11.4 [42] to simulate the delayed stochastic system. In this paper, the delayed reaction is not probabilistic, so after a delay of $\tau$ time units the delayed population decays to the product population with probability one. The inclusion of a probabilistic transition of the delayed population is possible but not considered here.

In the case of Gierer-Meinhardt kinetics (Sec. VF), oscillations occur which create huge increases in population size. Unfortunately, these increases are too big for the Gillespie SSA to handle practically. Thus, in cases where populations are too big to simulate explicitly, we use Langevin simulations akin to those introduced by Tian $e t$ al. [53]. The Langevin simulations are related to the time-explicit Euler stepping approach used in the deterministic simulations through the addition of two correctly scaled white noise terms which represent the stochasticity due to the present and delayed reactions, respectively. Langevin simulations are also used in the case of cubic autocatalytic kinetics with ligand internalization delays, since the time scales over which the simulations are needed to run cause the SSA to be impractical.

Finally, we must once again discuss the role of initial conditions; it is not simply enough to define the initial condition at time $t=0$. Due to the delay, we must also define the populations for the time bins during the period $-\tau \leqslant t<0$. Previously [26,54], these prior time points have simply been fixed at (small perturbations around) the steady state and, thus, products from the delayed equation are able to appear at the beginning of the simulation, $t=0$. Physically, these initial conditions model an experiment that is kept at steady state during the initial time period, $-\tau \leqslant t<0$. We choose to have the initial delayed concentrations set to zero and fix the $t=0$ condition to be the homogeneous steady state in the stochastic case and the homogeneous steady state with additional normally distributed random noise with mean 0 and standard deviation $1 / 10$ in the deterministic case. This small standard deviation was used to mimic the fact that the deterministic concentration is related to the stochastic population through the approximation $\phi+\eta_{u} / \sqrt{\Omega}=U / \Omega$ and $\psi+\eta_{v} / \sqrt{\Omega}=V / \Omega$, where the $\eta_{u}$ and $\eta_{v}$ are normally distributed random variables [37,45-49]. The value $\sigma=1 / 10$ comes from the fact that here, as elsewhere, we fix $\Omega=100$. The physical interpretation of these conditions is that prior to the start of the reactions the domain was empty, or reagents occupied the domain but were unable to react until $t=0$ when the reactants were uncaged.

Of course the reality of this initial condition may be contested as, if the reagents exist in the domain, then, unless they are constrained by some factor, they would surely react. However, in feather patterning there is a hypothesis of a priming wave [55,56], with an absence of patterning before the wave. If the reactions are primed in this way there are no reactions in the domain for $t<0$ and hence the conditions are not unreasonable.

\section{SIMULATIONS OF DELAYED KINETICS}

Having derived the delayed deterministic equations from the underlying reaction systems we compare the stochastic and 
deterministic simulations to assess whether stochastic effects are able to rescue the Turing mechanism from the concern that it is not physically plausible due to its sensitivity to kinetic delays.

\section{A. Schnakenberg ligand internalization simulations}

Deterministic and stochastic simulations of the ligand internalized system are shown in Fig. 3. First, we observe that, due to fixing the initial conditions to be zero for $-\tau \leqslant t<0$, and then at steady state for $t=0$, both the deterministic concentrations and stochastic populations suffer a large decrease from the steady-state populations lasting approximately $6 \tau$ time units. This is then followed by a large increase up to an order of 1000 particles per discretized interval. Finally, the huge population oscillation decreases to the steady state and, from there, progression to the patterned state occurs.

Further, we see that, at least for these parameter values, although noise is able to expedite pattern formation, the Schnakenberg kinetics with ligand internalization are incredibly sensitive to small changes (in relation to the time scale of pattern formation) in the kinetic delays. Thus, due to this temporal sensitivity induced by the delay, we conclude that this form of kinetics is likely to be unsuitable for rapid, temporally regulated developmental systems, such as those found in zebrafish [8].
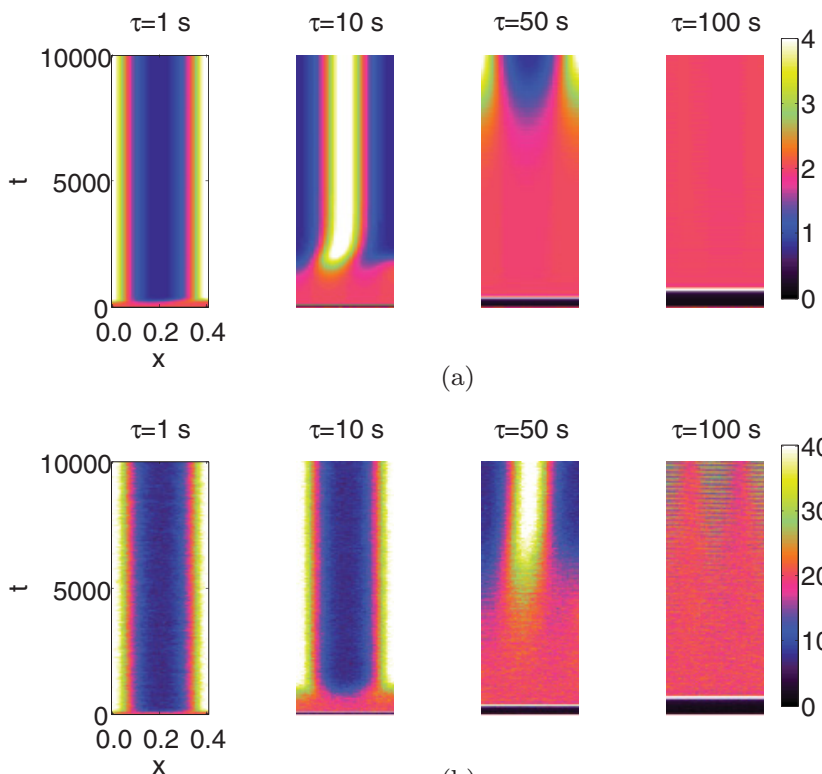

(a)
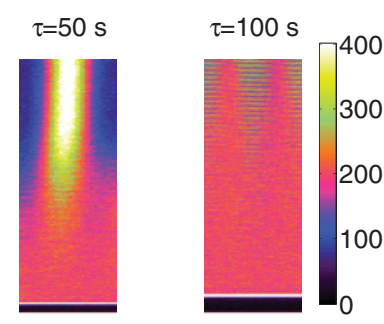

(b)

FIG. 3. (Color online) Top: concentration $\phi$ of the delayed deterministic Schnakenberg system with ligand internalization, Eqs. (11) and (12), simulated using a modified explicit Euler stepping method. Bottom: population $U$ of the delayed stochastic Schnakenberg system, simulated using Dizzy 1.11.4 [42]. The delay, $\tau$, in seconds, is given above each figure. All of the images inherit the left-hand axes and the right-hand color map. Parameters are $D_{u}=$ $10^{-4} \mathrm{~mm}^{2} / \mathrm{s}, D_{v}=10^{-2} \mathrm{~mm}^{2} / \mathrm{s}, c_{1}=0.1 \mathrm{~mol} /(\mathrm{mm} \mathrm{s}), c_{-1}=0.2 / \mathrm{s}$, $c_{2}=0.3 \mathrm{~mol} /(\mathrm{mm} \mathrm{s})$, and $c_{3}=0.1 \mathrm{~mm} /\left(\mathrm{mol}^{2} \mathrm{~s}\right)$.
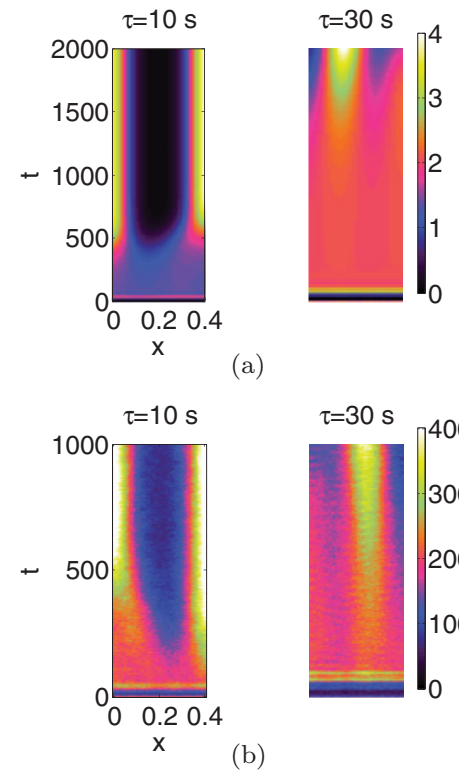

FIG. 4. (Color online) Top: concentration $\phi$ of the delayed deterministic Schnakenberg system with reversible-ligand binding, Eqs. (8) and (9). Bottom: population $U$ of the delayed stochastic Schnakenberg system. The delay, $\tau$, in seconds, is given above each figure. All of the images inherit the left-hand axes and the right-hand color map. The parameters and solution methods are the same as in Fig. 3.

\section{B. Schnakenberg reversible-ligand binding simulations}

We now consider pattern initiation for the reversible-ligand binding form of delay. From Fig. 4 we can immediately see that this other form of delay can produce a large difference in the simulated outcome. Once again, we see that initially the population drops and then undergoes a large increase. After this the population tends to the steady state and finally the pattern is produced from this state. Although these dynamics are attributable to both ligand internalization and reversibleligand binding forms of delay (compare Figs. 3 and 4), in the current case of reversible-ligand binding these initial transient effects are not as sensitive to the delay as in the case of ligand internalization.

By comparing Figs. 4(a) and 4(b) we see that stochasticity is able to remove the patterning lag that is seen in the deterministic simulations. Explicitly, in Fig. 4(a) we observe that the patterning lag is initially $600 \mathrm{~s}$ for a delay of $\tau=10 \mathrm{~s}$. This then slowly increases to a lag of $1500 \mathrm{~s}$, with the increase of the delay up to $\tau=30 \mathrm{~s}$. However, in the case of the stochastic system, the pattern is always seen to be initiated within $1000 \mathrm{~s}$.

Knowing that noise helps the system produce patterns earlier, we increased the delay further to see how far we could push the system before it began to break down. In Fig. 5 we see that even for a delay of $\tau=100 \mathrm{~s}$ Turing patterns are observable. Critically, although it takes roughly 4000-5000 s for the patterns to stabilize, we can see that the initialization occurs at around $1000 \mathrm{~s}$. If the delay is then pushed even further spatial heterogeneity is still visible over a long time scale, although it is now accompanied by oscillations that have a consistent frequency. By considering higher delays (data not 


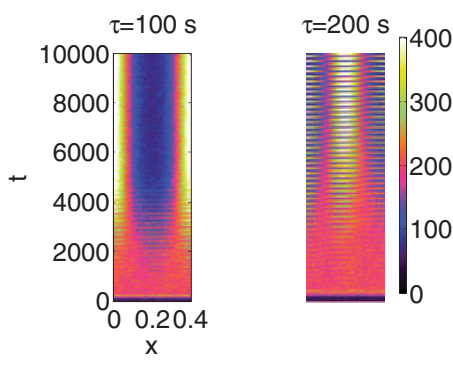

FIG. 5. (Color online) Population $U$ of the delayed stochastic Schnakenberg system with reversible-ligand binding, Eq. (7). The delay, $\tau$, in seconds, is given above each figure. All of the images inherit the left-hand axes and the right-hand color map. The parameters and solution methods are the same as in Fig. 3.

shown) the time scale of the oscillations is observed to increase along with the delay.

\section{Glycolysis ligand internalization simulations}

The effect of the ligand internalization delays on the glycolysis reactions is demonstrated in Fig. 6 and is very similar to those of the Schnakenberg kinetics. However, although the patterning initiation time still depends on the delay, $\tau$, its sensitivity is greatly reduced as we can increase
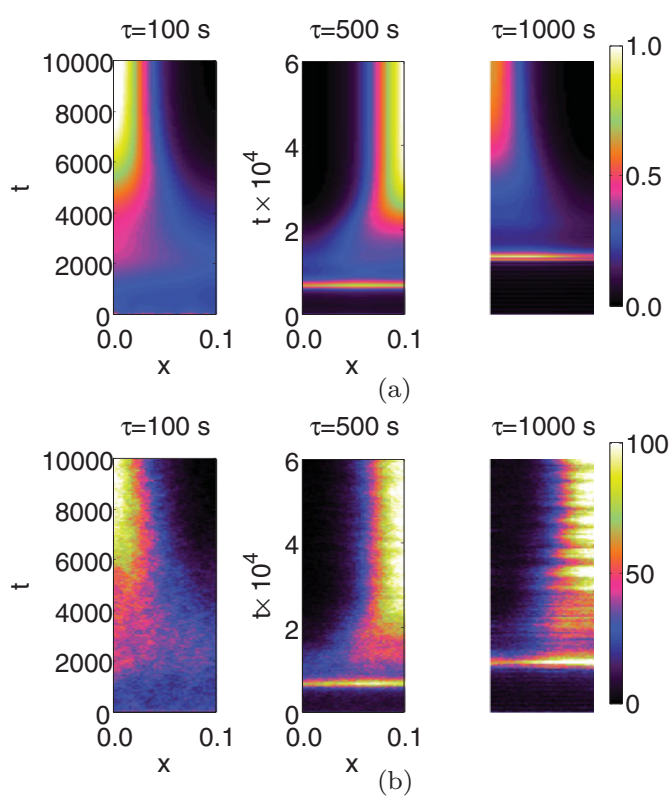

FIG. 6. (Color online) Top: concentration $\psi$ of the delayed deterministic glycolysis system with ligand internalization, Eqs. (15) and (16), simulated using a modified explicit Euler stepping method. Bottom: population $V$ of the delayed stochastic glycolysis systems, simulated using Dizzy 1.11.4 [42]. The delay, $\tau$, in seconds, is given above each figure. The $\tau=500$ and $1000 \mathrm{~s}$ images are plotted on the same axes. All of the images inherit the right-hand color map. Parameters are $c_{1}=4.8 \times 10^{-3} \mathrm{~mol} /(\mathrm{mm} \mathrm{s}), c_{2}=8 \times 10^{-5}$ $\mathrm{s}, c_{3}=0.04096 \mathrm{~mm} /\left(\mathrm{mol}^{2} \mathrm{~s}\right), c_{4}=1.25 \times 10^{7} \mathrm{~s}, c_{5}=1.6 \times 10^{-3}$ $\mathrm{s}, c_{6}=5.12 \times 10^{-3} \mathrm{~mm} /\left(\mathrm{mol}^{2} \mathrm{~s}\right), D_{\phi}=10^{-4} \mathrm{~mm}^{2} / \mathrm{s}$, and $D_{\psi}=$ $3 \times 10^{-7} \mathrm{~mm}^{2} / \mathrm{s}$. the kinetic delay far beyond that of the Schnakenberg system and still induce patterning on significantly shorter time scales.

The effects of noise on these kinetics are seen to be minimal in that the pattern initiation time is comparable to that of the deterministic simulation. In certain cases (data not shown) it has been observed that the intrinsic stochasticity causes the initiation to be quite noisy and, thus, although the mode one wave has been activated, the analogous deterministic simulation is able to produce a clearer pattern much earlier.

\section{Glycolysis reversible-ligand binding simulations}

In Fig. 7 we see that the behavior of the glycolysis kinetics is similar to Schnakenberg kinetics in that the systems are less sensitive to the delay when reversible-ligand binding delays are used than when ligand internalization delays are used. Moreover, the glycolysis reactions are able to withstand much higher kinetic delays compared to the Schnakenberg kinetics. Indeed, by pushing the delay further to our computational limits (simulations not shown) we observe that, unlike the Schnakenberg kinetics, the kinetics never appear to break down to constant oscillations. There may be initial oscillations, as shown in the $\tau=3000 \mathrm{~s}$ simulations, but these are always damped leaving a final stable steady state.

Similar to the glycolysis simulations with ligand internalization the effects of noise on the glycolysis reactions appear to be minimal as in the majority of cases the pattern initiation time is equivalent in both sets of simulations. However, it is noted that in the stochastic $\tau=3000 \mathrm{~s}$ case, although the initiation of a pattern is observable during the temporal interval
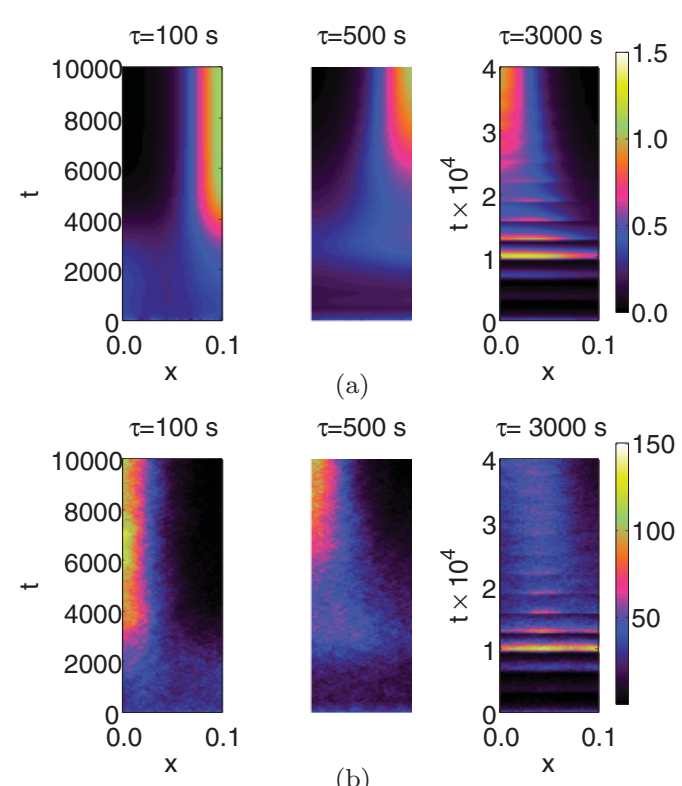

(b)

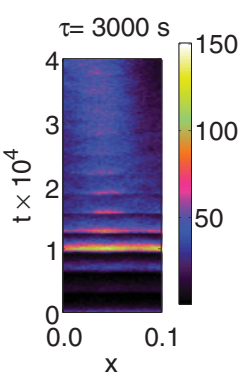

FIG. 7. (Color online) Top: concentration $\psi$ of the delayed deterministic glycolysis system with reversible-ligand binding, Eqs. (13) and (14), simulated using a modified explicit Euler stepping method. Bottom: population $V$ of the delayed stochastic glycolysis system. The delay, $\tau$, in seconds, is given above each figure. All figures are plotted on the same axes except for the $\tau=3000 \mathrm{~s}$ images where the axes are shown explicitly. All of the images inherit the right-hand color map. The parameters and solution methods are the same as in Fig. 6. 

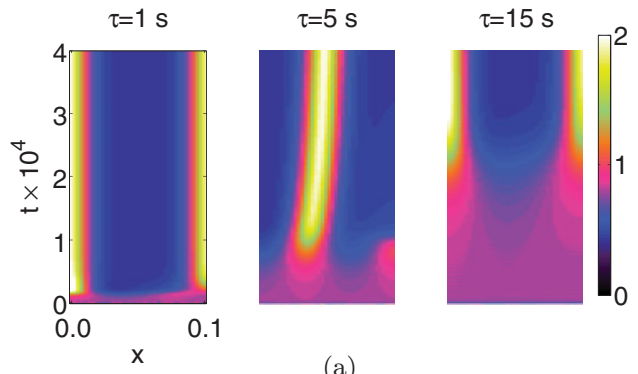

(a)
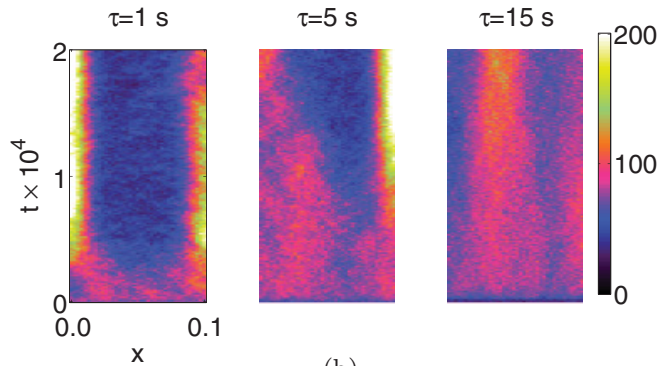

(b)

FIG. 8. (Color online) Top: concentration $\phi$ of the delayed deterministic Gierer-Meinhardt system with ligand internalization, Eqs. (19) and (20), simulated using a modified explicit Euler stepping method. Bottom: population $U$ of the delayed stochastic Gierer-Meinhardt system, simulated using Dizzy 1.11.4 [42]. The delay, $\tau$, in seconds, is given above each figure. All of the images inherit the left-hand axes and the right-hand color map. Parameters are $D_{\phi}=3.125 \times 10^{-6} \mathrm{~mm}^{2} / \mathrm{s}$, $D_{\psi}=2.5 \times 10^{-3} \quad \mathrm{~mm}^{2} / \mathrm{s}, \quad c_{1}=0.06 \quad \mathrm{~mol} /(\mathrm{mm} \quad \mathrm{s})$, $c_{2}=0.2 \mathrm{~s}, c_{3}=0.125 \mathrm{~s}, c_{4}=1.25 \mathrm{~mm} /(\mathrm{mol} \mathrm{s})$, and $c_{5}=0.1 \mathrm{~s}$.

$2 \times 10^{4} \mathrm{~s}<t<4 \times 10^{4} \mathrm{~s}$, it is not as clear as its deterministic analog.

\section{E. Gierer-Meinhardt ligand internalization simulations}

Once again, we begin by considering the effects of ligand internalization delays. The stochastic and deterministic simulations are shown in Fig. 8. Essentially, the results produced for these kinetics with ligand internalization delay are very similar to those in the case of the Schnakenberg kinetics, except that the pattern formation lag is even more severe. Once again, stochasticity appears to aid in reducing this lag, as can be seen in the $\tau=15$ s case of Fig. 8, but, on the whole, the pattern initiation time scale is too sensitive to small changes in the kinetic delay for the system to be of any practical use.

\section{F. Gierer-Meinhardt reversible-ligand binding simulations}

We now consider the simulation results of the reversibleligand binding form of delay shown in Fig. 9. Here, we observe that for this system even small delays can cause a complete breakdown of Turing patterning. In the deterministic case, Fig. 9(a), the spatial inhomogeneity is replaced with homogeneous oscillations, while in the stochastic cases, Figs. 9(b) and 9(c), inhomogeneous oscillations are seen. Note that this breakdown is not a numerical error as the simulations are unaffected by a decrease in the time step or increase in the number of time bins. Also, these effects have been observed and described previously in deterministic systems [27].
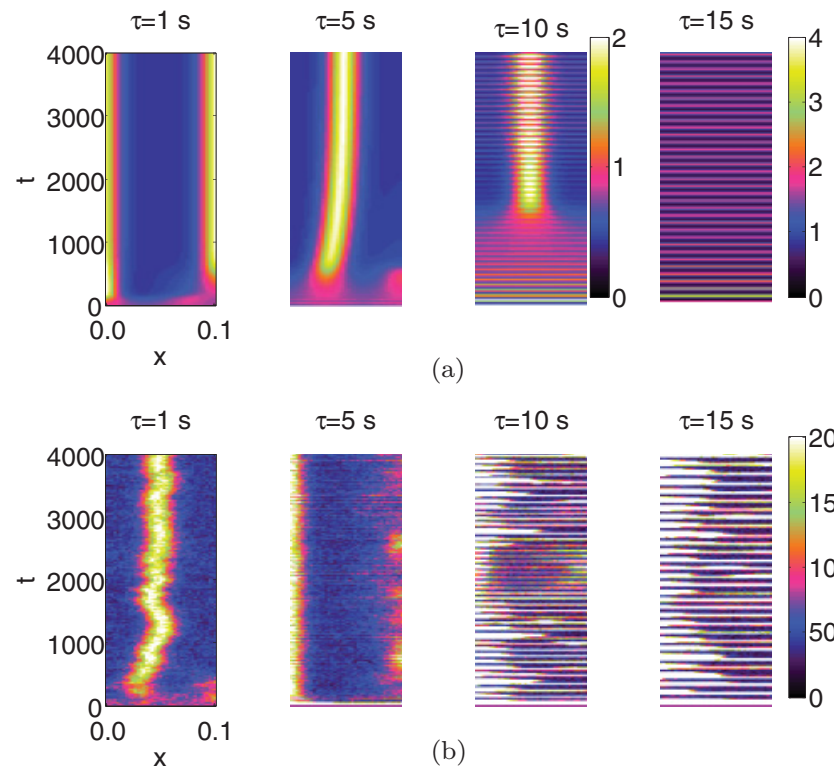

(a)
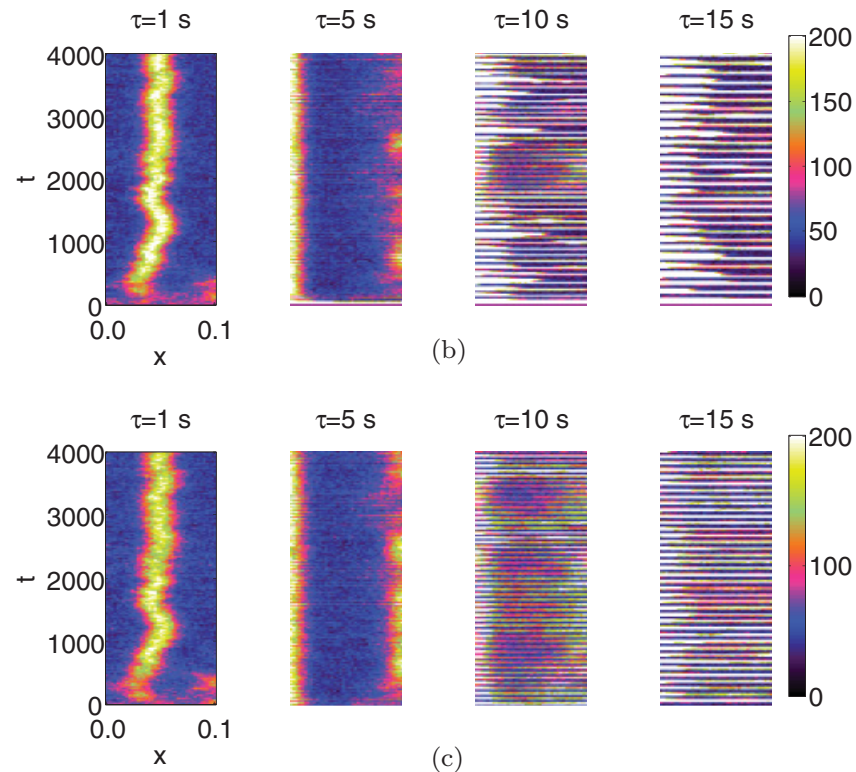

(b)
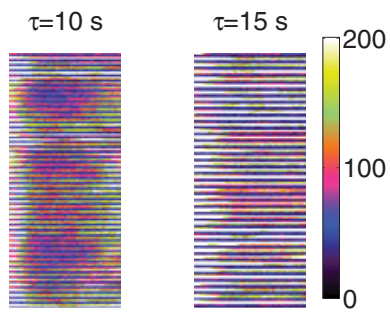

(c)

FIG. 9. (Color online) Top: concentration $\phi$ of the delayed deterministic Gierer-Meinhardt system with reversible-ligand binding, Eqs. (17) and (18), simulated using a modified explicit Euler stepping method. Middle: population $U$ of the delayed stochastic GiererMeinhardt system, simulated using a Langevin form of Eqs. (17) and (18) [53]. Bottom: population $U$ of the delayed stochastic Gierer-Meinhardt system with saturation, simulated using a Langevin form of Eqs. (17) and (18), where the nonlinear term in Eq. (17) has been modified to Eq. (25). In both the middle and bottom images, the color axes has been capped at 200 particles. However, the populations can increase to levels much greater than this. The delay, $\tau$, is given above each figure in seconds. All of the images inherit the left-hand axes and the color map is either given for each image or is inherited from the right. The parameters are the same as in Fig. 8.

Due to the oscillations causing huge increases in the populations, we are computationally unable to simulate the stochastic systems using the standard Gillespie SSA. Thus, instead, Figs. 9(b) and 9(c) are simulated using a Langevin method, as noted in Sec. IV. Further, in order to try and reduce this population increase, the simulations in Fig. 9(c) have a modified nonlinear term,

$$
\frac{\kappa_{3} U^{2}}{\left(1+10^{-6} U^{2}\right)(V+0.001)},
$$

causing the kinetics to saturate. Delays are included within this term as previously described.

Although the main dynamics observed in the deterministic simulations [Fig. 9(a)] are recapitulated in the stochastic simulations [Figs. 9(b) and 9(c)], we immediately see that noise 
actually has a destructive effect on the Turing mechanism. Specifically, in the case of $\tau=5 \mathrm{~s}$, the Turing patterns in the noisy environments are much weaker than their deterministic analogues, in that they frequently break up and disappear. Equally, in the oscillating cases, $\tau \geqslant 10 \mathrm{~s}$, the oscillations are seen to be extremely irregular, with the populations increasing far above the values seen in the deterministic simulation, as, although the color map in Figs. 9(b) and 9(c) is capped at 200 particles, the populations reach levels of $10^{8}$ and $10^{5}$ particles, respectively.

Thus we see that, not only is the breakdown to oscillations for small delay values a key difference between the pure and cross kinetics we have studied, their responses to intrinsic noise are also different. By increasing the population scale, $\Omega$, we could reduce the effects of the noise on the Gierer-Meinhardt system but, critically, for the same order of particle numbers intrinsic noise appears to have a less destructive influence on the cross kinetics.

\section{G. Cubic autocatalysis ligand internalization simulations}

The simulations for the cubic autocatalysis kinetics with ligand internalization, Eqs. (23) and (24), are shown in Fig. 10 and by comparing this figure to Figs. 8,9 , and 11 we are able to make the observation that the delays do not affect the Gierer-Meinhardt system in the same way as the cubic autocatalysis system, even though they are both pure kinetics.
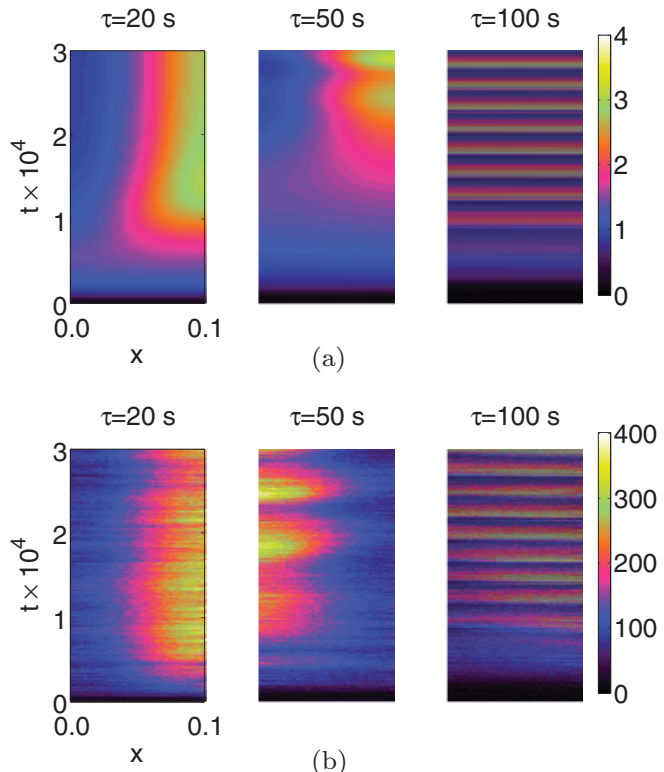

(b)

FIG. 10. (Color online) Top: concentration $\phi$ of the delayed deterministic cubic autocatalytic system with ligand internalization, Eqs. (23) and (24), simulated using a modified explicit Euler stepping method. Bottom: population $U$ of the delayed stochastic cubic autocatalytic system, simulated using a Langevin form of Eqs. (23) and (24). The delay, $\tau$, in seconds, is given above each figure. All of the images inherit the left-hand axes and the right-hand color map. Parameters are $c_{1}=0.06 \mathrm{~s}, c_{2}=0.02 \mathrm{~s}, c_{3}=0.1 \mathrm{~mm} /(\mathrm{mol}$ $\mathrm{s}), c_{4}=0.0061 \mathrm{~mol} /(\mathrm{mm} \mathrm{s}), c_{5}=0.002 \mathrm{~s}, c_{6}=0.0222 \mathrm{~s}, c_{7}=$ $0.0301 \mathrm{~mm} /(\mathrm{mol} \mathrm{s}), c_{8}=0.01 \mathrm{~mm}^{2} /\left(\mathrm{mol}^{2} \mathrm{~s}\right), D_{\phi}=10^{-4} \mathrm{~mm}^{2} / \mathrm{s}$, and $D_{\psi}=10^{-6} \mathrm{~mm}^{2} / \mathrm{s}$.
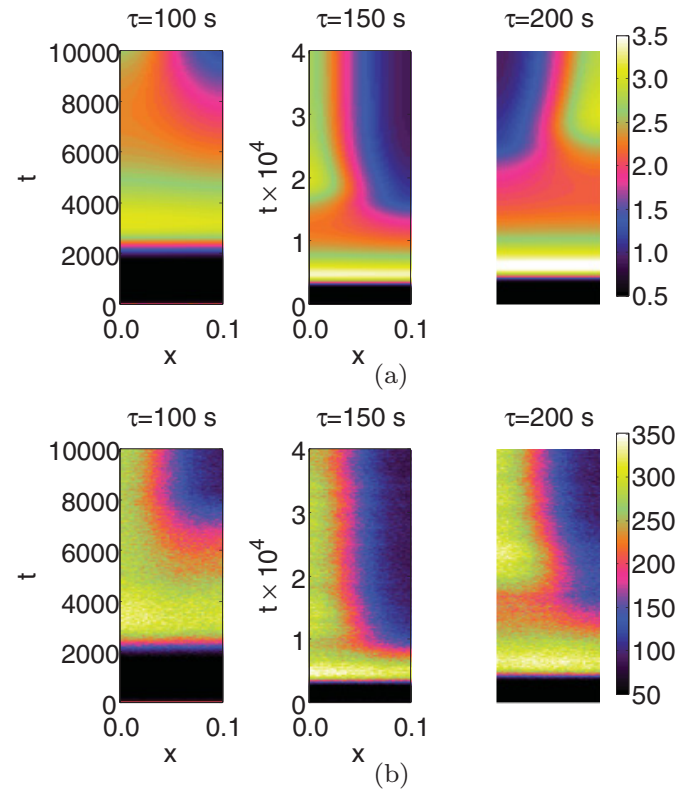

FIG. 11. (Color online) Top: concentration $\phi$ of the delayed deterministic cubic autocatalytic system with reversible-ligand binding, Eqs. (23) and (24). Bottom: population $U$ of the delayed stochastic cubic autocatalytic system. The delay, $\tau$, in seconds, is given above each figure. Images $\tau=150$ and $200 \mathrm{~s}$ have the same axes. The axes for each image is either given or inherited from their immediate left and the color map is inherited from the right-hand side. The parameters and solution methods are the same as in Fig. 10.

Primarily, we notice that it is the ligand internalization delay that causes this system to break down to oscillations, rather than reversible-ligand binding delays. However, both forms of pure kinetics do have the similarity that they are extremely sensitive to the kinetic delay and, as such, are unsuitable for general biological purposes, particularly where regulation over time is also required.

When comparing deterministic and stochastic simulations we see that the noise is able to initiate the patterns earlier [Fig. 10(b)] than those observed in Fig. 10(a). However, not even the noise can stop the breakdown of patterning to oscillations for relatively small values of $\tau$.

\section{H. Cubic autocatalysis reversible-ligand binding simulations}

Finally, we deterministically and stochastically simulate the cubic autocatalysis kinetics with reversible-ligand binding simulations, Eqs. (23) and (24), the results of which are shown in Fig. 11. Although the simulations have not been observed to break down to oscillations as in the ligand internalization case in the previous section, we see that the patterning lag is still sensitive to alterations in the kinetic delay.

However, noise does appear to aid in reducing the time to pattern initiation, as can be seen by comparing Figs. 11(a) 
and 11(b). In particular, for $\tau=100 \mathrm{~s}$, the deterministic pattern does not appear until around $9000 \mathrm{~s}$, whereas the noisy simulations can exhibit heterogeneity within approximately $7000 \mathrm{~s}$. Similarly, when $\tau=150$ and $200 \mathrm{~s}$, the deterministic simulations produce patterns around $5000 \mathrm{~s}$ slower than their stochastic counterparts.

\section{CONCLUSION}

We started by demonstrating that the patterning initiation time in Turing systems can be reduced through the addition of noise. This was then used to motivate investigations into the effects of delays on stochastic patterning systems as their deterministic analogues have been shown to produce some extremely anomalous behavior. Our interest in adding delays to the system is not simply an interesting mathematical abstraction as cellular systems, to which we apply such reaction-diffusion equations, go through a complex procedure of producing RNA and mRNA before producing the relevant proteins. Overall, protein production delays can be anywhere from several minutes to hours [18,19].

It should be noted that the results in this paper have not been compared to predictions of linear theory as it has been shown that, in general, linear analysis of delayed reaction-diffusion equations is not reliable [26]. However, through completing the standard Turing analysis for nondelayed systems (not shown) we can be sure that in each case the stochastic simulations do evolve to a wave mode that is consistent with the linearized spectrum.

As the exact form of delay in such biological systems is unknown we concentrated on simulating the effects of two extreme, but experimentally motivated, forms of delay: ligand internalization, which models a cell engulfing the ligand; and reversible-ligand binding, where the ligands are able to return to the bulk population as soon as they have initiated production of the final product. We have shown that their effects on both the stochastic and deterministic systems are quite different. This indicates that if delays are to be included in a system it is imperative that the correct form of delay be justified from the application.

In order to observe the interaction of noise and delays in Turing systems we simulated both delay forms with four different kinetics, two of a pure type and two of a cross type. For each set of kinetics we simulated ligand internalization delays and compared these to the simulations of reversible-ligand binding delays. Immediately, we saw that all kinetics suffered from greater patterning lags and temporal sensitivity due to ligand internalization, when compared to reversible-ligand binding. Note that this increased sensitivity to ligand internalization is present in both the deterministic and stochastic formalisms, for although in some cases, e.g., cubic autocatalysis kinetics [see Fig. 10(b)], noise is seen to initiate the patterns earlier, the pattern initiation time scale is still large compared to the size of the kinetic delay. Nonetheless, the glycolysis kinetics were still able to pattern over biologically relevant time scales for relatively large delays.

Due to the great variation in time scales displayed by the different Turing kinetics we must be careful when applying such systems to biological problems. In situations where development is slow $[16,57]$ we perhaps do not need not worry about small kinetic delays greatly amplifying patterning lags. In such cases delays only cause a problem when they are able to cause a complete loss of spatial heterogeneity, which is seen using Gierer-Meinhardt kinetics with reversible-ligand binding, Fig. 9(c), and cubic autocatalysis kinetics with ligand internalization, Fig. 10(b). However, in rapidly developing systems $[4,8]$ it is much more difficult to justify the use of Turing kinetics due to their sensitivity to delays.

In the case of Schnakenberg kinetics intrinsic stochasticity is seen to reduce the patterning time in both ligand internalization and reversible-ligand binding cases. However, the pattern formation time is only reduced to a feasible level in the reversible-ligand binding case as it is less sensitive to the kinetic delay. Problematically, if the reversible-ligand binding delay is increased too far, we see that the steady state breaks down to spatially heterogeneous oscillations. This motivates a brief discussion about biologically feasible time scales. In this paper the parameters have been defined in terms of seconds. Since the delays can occur on a time scale of minutes, i.e., $\tau \sim$ $100 \mathrm{~s}$, we would be toward the upper end of the possible range over which the Schnakenberg kinetics can still realize patterns. However, it has been shown that reversible-ligand binding can also occur on the order of minutes [58]. Thus, if we rescale all of the parameters to be on the size of minutes instead of seconds, e.g., $0.1 \mathrm{~mol} / \mathrm{s} \mapsto 0.1 \mathrm{~mol} / \mathrm{min}$, we greatly increase the range of delays over which the stochastic Schnakenberg system can pattern in a reasonable time.

Similarly, the second form of cross kinetics that we considered, the glycolysis kinetics, were also much less influenced by the kinetic delay when encoded through reversible-ligand binding. Further, over the parameter region that we considered, the glycolysis kinetics never break down to constant oscillations as in the Schnakenberg case. However, unlike the Schnakenberg system in which noise did decrease pattern initiation time we see that stochasticity has minimal effects on the glycolysis kinetics. This relative insensitivity to stochasticity and delays implies that the glycolysis kinetics are a viable candidate for patterning in deterministic systems, especially over shorter time scales. Furthermore, we clearly see that the effect of noise on a reaction-diffusion system is heavily dependent on the kinetics. This difference in behavior is useful when distinguishing which kinetic systems are appropriate for particular applications.

The effects of noise on the pure kinetics were similarly varied. Critically, the ligand internalization Gierer-Meinhardt system was extremely sensitive to the delays, suggesting that some other mechanisms would be needed for it to be a viable candidate for patterning on rapid developmental time scales. Further, although pattern initiation in the reversible-ligand binding system was much less sensitive to small ranges of the kinetic delay, if this delay is increased slightly spatially stable pattern formation is lost in both the deterministic and stochastic simulations and replaced with large oscillations. In contrast to the cross kinetics with reversible-ligand binding previously discussed, not only does noise not help reduce the patterning delay in the reversible-ligand binding GiererMeinhardt system, it also acts as a destructive mechanism, causing the Turing patterns to break down for long periods of time. Equally, noise also has a negative effect on the regularity of the oscillations, whereas in the large delay stochastic 
Schnakenberg kinetic case (Fig. 5) the oscillation frequency is much more consistent.

Finally, although the cubic autocatalysis kinetics display similar dynamics to the Gierer-Meinhardt kinetics the effects of the different delays appear to be reversed. Specifically, it is ligand internalization delays that cause the oscillations in the cubic autocatalysis kinetics, whereas it is reversibleligand binding delays that produce oscillations in the GiererMeinhardt system. Moreover, it is observed that the oscillations in the stochastic simulations of the cubic autocatalysis kinetics are much more regular and consistent with their deterministic simulation than the Gierer-Meinhardt kinetics, once again illustrating that kinetics with a similar theoretical basis need not behave in the same way once delays are included. Similar to the rest of the investigated kinetics, the cubic autocatalysis system shows an increased temporal sensitivity to ligand internalization delays, when compared to reversible-ligand binding delays. Equally, similar to the glycolysis kinetics, although intrinsic stochasticity is able to reduce the time to pattern formation slightly, the effect is generally minimal.

It has been noted previously [27] that delays can affect pure and cross kinetics differently. Here, we have shown that exactly the same is true in the case of stochastic kinetics and, moreover, the effects of noise on these different cases is also observed to vary. These differences between stochastic and deterministic frameworks, ligand internalization and reversible-ligand binding descriptions of delay, and pure and cross kinetic systems all aid the construction of biologically realistic models, as these insights allow us to distinguish the most appropriate mathematical components for specific applications.

In summary, in relation to the chosen kinetic parameter values, if one is not dealing with relatively rapid developmental systems the observed sensitivity to delays may be tolerable. However, in the case in which developmental time is fast we are able to suggest that, although delays are able to produce aberrant effects in deterministic reaction-diffusion systems, there are certain specific conditions, e.g., glycolysis kinetics with reversible-ligand binding, where pathologies in delayed Turing systems are ameliorated. This contrasts with the conclusions previously drawn $[26,28,54,59]$ emphasizing the structural sensitivity of delayed Turning patterning. In addition, there are also conditions under which stochasticity is able to act against the dominant dynamics, thereby impairing the pattern formation mechanism (see Sec. V F). Due to these vastly different effects of noise it would be interesting to further investigate delayed stochastic systems by simulating them on growing domains. Although stochastic systems on growing domains may not be able to generate robust transition sequences, we might speculate that the noise could disrupt the homogeneous steady state and initiate pattern formation, thus removing the pattern breakdown which has been observed in the deterministic case [54,59].

\section{ACKNOWLEDGMENTS}

T.E.W. would like to thank the EPSRC for support. P.K.M. was partially supported by a Royal Society-Wolfson Research Merit Award. This publication was based on work supported in part by Award No. KUK-C1-013-04, made by King Abdullah University of Science and Technology (KAUST).

\section{APPENDIX A: KINETICS}

In this Appendix we provide the reactions that form the basis of the glycolysis, Gierer-Meinhardt and cubic autocatalysis systems.

Glycolysis. The reactions are

$$
\begin{aligned}
\emptyset \stackrel{\kappa_{1}}{\rightarrow} U, \quad U \stackrel{\kappa_{2}}{\rightarrow} \emptyset, \quad 2 V+U \stackrel{\kappa_{3}}{\rightarrow} 2 V, \quad U \stackrel{\kappa_{4}}{\rightarrow} U+\underset{(\mathrm{A} 1)}{V,} \\
V \stackrel{\kappa_{5}}{\rightarrow} \emptyset, \quad 2 V+U \stackrel{\kappa_{6} \text {,elay } \tau}{\longrightarrow} 3 V .
\end{aligned}
$$

The reversible-ligand binding delayed reaction is

$$
2 V+U \stackrel{\kappa_{6}}{\rightarrow} 2 V+W, \quad W \stackrel{\text { delay } \tau}{\longrightarrow} V .
$$

The ligand internalization delayed reaction is

$$
2 V+U \stackrel{\kappa_{6}}{\rightarrow} W, \quad W \stackrel{\text { delay } \tau}{\longrightarrow} 3 V .
$$

Gierer-Meinhardt system. The reactions are

$$
\begin{gathered}
\emptyset \stackrel{\kappa_{1}}{\rightarrow} U, \quad U \stackrel{\kappa_{2}}{\rightarrow} \emptyset, \quad V \stackrel{\kappa_{5}}{\rightarrow} \emptyset, \quad 2 U \stackrel{\kappa_{3} /(V+0.001) \text { delay } \tau}{\longrightarrow} 3 U, \\
2 U \stackrel{\kappa_{4} \text { delay } \tau}{\longrightarrow} 2 U+V .
\end{gathered}
$$

The reversible-ligand binding delayed reactions are

$$
\begin{array}{r}
U+U \stackrel{\kappa_{3} /(V+0.001)}{\longrightarrow} W_{1}+U+U, \quad W_{1} \stackrel{\text { delay } \tau}{\longrightarrow} U, \\
U+U \stackrel{\kappa_{4}}{\rightarrow} W_{2}+U+U, \quad W_{2} \stackrel{\text { delay } \tau}{\longrightarrow} V .
\end{array}
$$

The ligand internalization delayed reactions are

$$
\begin{aligned}
& U+U \stackrel{\kappa_{3} /(V+0.001)}{\longrightarrow} W_{1}, \quad W_{1} \stackrel{\text { delay } \tau}{\longrightarrow} 3 U, \\
& U+U \stackrel{\kappa_{4}}{\rightarrow} W_{2}, \quad W_{2} \stackrel{\text { delay } \tau}{\longrightarrow} U+U+V .
\end{aligned}
$$

Cubic autocatalytic system. The reactions are

$$
\begin{gathered}
U \stackrel{\kappa_{1}}{\rightarrow} \emptyset, \quad 2 U \stackrel{\kappa_{2}}{\rightarrow} U, \quad \emptyset \stackrel{\kappa_{4}}{\rightarrow} V, \quad V \stackrel{\kappa_{5} U / V}{\rightarrow} \emptyset, \\
V \stackrel{\kappa_{6}}{\rightarrow} \emptyset, \quad 3 V \stackrel{\kappa_{8}}{\rightarrow} 2 V, \\
V+U \stackrel{\kappa_{3}, \text { delay } \tau}{\longrightarrow} 2 U+V, \quad 2 V \stackrel{\kappa_{7} \text {, delay } \tau}{\longrightarrow} 3 V .
\end{gathered}
$$

The reversible-ligand binding delayed reactions are

$$
\begin{gathered}
V+U \stackrel{\kappa_{3}}{\rightarrow} W_{1}+V+U, \quad W_{1} \stackrel{\text { delay } \tau}{\longrightarrow} U, \\
2 V \stackrel{\kappa_{7}}{\rightarrow} W_{2}+2 V, \quad W_{2} \stackrel{\text { delay } \tau}{\longrightarrow} V .
\end{gathered}
$$

The ligand internalized form of the delayed reactions is

$$
\begin{aligned}
& V+U \stackrel{\kappa_{3}}{\rightarrow} W_{1}, \quad W_{1} \stackrel{\text { delay } \tau}{\longrightarrow} 2 U+V, \\
& 2 V \stackrel{\kappa_{7}}{\rightarrow} W_{2}, \quad W_{2} \stackrel{\text { delay } \tau}{\longrightarrow} 3 V .
\end{aligned}
$$

\section{APENDIX B: NUMERICAL ALGORITHM}

To simulate the deterministic system we use an explicit Euler stepping algorithm [52], which updates the concentrations 


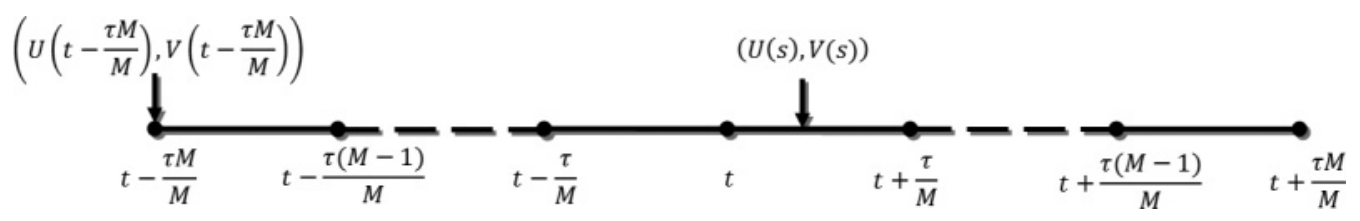

FIG. 12. Illustration of the time axis discretized into bins. See text for details.

at discrete time points based on a time step, $d t$, that is an input of the algorithm. In order to satisfy numerical stability of the algorithm $d t$ is bounded above. However, we are able to make $d t$ as small as computationally feasible [53]. Due to this certain amount of freedom of choice over $d t$ we are able to choose it such that it is an integer divisor of $\tau / M$. Hence, as the concentrations are calculated at integer multiples of $d t$, we can determine the concentration at the start of each time bin. Unfortunately, we do not have this form of control in the case of the Gillespie algorithm as the time step is computed as an integral part of the SSA. Thus we fix the delayed population of a time bin to be the population at time $t^{\prime}$, where $t^{\prime}$ is the first time point to cross the time bin's threshold. If $M$ is chosen to be large this should produce minimal numerical differences in the simulation, and we have verified that our results do not change on further increases in $M$.

Suppose we are interested in outputting the value of the populations at time points $0, T, 2 T$, etc. Since we also need to keep track of $\tau$ time units worth of history, the simulation time axis is broken up into steps of size $\tau / M$. This idea is illustrated in Fig. 12 where we consider a specific time point $t=n T$ for some $n \in \mathbb{Z}$. In the stochastic case, the population at the next time point, $\left[U\left(s^{\prime}\right), V\left(s^{\prime}\right)\right]$, will be calculated through kinetics involving the current populations, $[U(s), V(s)]$, and the past populations, $[U(t+\alpha \tau / M-\tau), V(t+\alpha \tau / M-\tau)]$, where $\alpha$ is the integer satisfying $t+\alpha \tau / M \leqslant s<t+(\alpha+1) \tau / M$. Further, as time steps forward, with maximum step $d t \ll \tau / M$, the populations $[U(t+z \tau / M), V(t+z \tau / M)]$ are recorded for $z=0, \ldots, M$ and are used as the delayed populations in the next $\tau$ time step. Note that the initial $M$ populations for time $-\tau \leqslant t \leqslant 0$ have to be specified, but, after this, all of the populations are generated as the system evolves. The deterministic simulations are constructed in exactly the same way except that we use the concentrations $(\phi, \psi)$ instead of the populations $(U, V)$.

The algorithms do not resolve time within each time bin, $[t+\alpha \tau / M, t+(\alpha+1) \tau / M]$, as the populations $[U(s), V(s)]$ (concentrations $[\phi(s), \psi(s)]$ ) are used in conjunction with the past population $[U(t+\alpha \tau / M-\tau), V(t+\alpha \tau / M-\tau)]$ $([\phi(t+\alpha \tau / M-\tau), \psi(t+\alpha \tau / M-\tau)])$. For mathematical exactness, the delayed populations should be $[U(s-\tau), V(s-\tau)] \quad([\phi(s-\tau), \psi(s-\tau)]) . \quad$ However, by taking $M$ large enough we simply incur discretization errors at the same level of standard first order numerical algorithms. Hence $M$ is chosen on an ad hoc basis, through simulating the system with one value of $M$ and then increasing it by a factor of 10 until changes in $M$ stopped producing different effects in the simulation. Through this we have chosen the following values: $M=1000$ when $\tau \leqslant 100$; $M=10000$ when $100<\tau \leqslant 1000$; and $M=30000$ when $\tau=3000$. Note that if $M$ is taken large enough then the stochastic algorithm would effectively be capturing every single reaction that occurs and thus it would no longer be an approximate method.
[1] L. Wolpert, J. Theor. Biol. 25, 1 (1969).

[2] E. H. Davidson, Gene Activity in Early Development (Academic Press, New York, 1986).

[3] S. C. Stearns, Bioscience 39, 436 (1989).

[4] M. Towers, J. Signolet, A. Sherman, H. Sang, and C. Tickle, Nat. Commun. 2, 426 (2011).

[5] M. Towers, R. Mahood, Y. Yin, and C. Tickle, Nature (London) 452, 882 (2008).

[6] L. Wolpert, Towards a Theoretical Biology. I. Prolegomena (Edinburgh University Press, Edinburgh, 1968), Vol. 1, Chap. 10, pp. 125-133.

[7] J. Massague, Annu. Rev. Cell. Biol. 6, 597 (1990).

[8] C. B. Kimmel, W. W. Ballard, S. R. Kimmel, B. Ullmann, and T. F. Schilling, Am. J. Anat. 203, 253 (1995).

[9] A. M. Turing, Philos. Trans. R. Soc. London B 237, 37 (1952).

[10] R. Sakuma, Y. Ohnishi, C. Meno, H. Fujii, H. Juan, J. Takeuchi, T. Ogura, E. Li, K. Miyazono, and H. Hamada, Genes Cells 7, 401 (2002).

[11] L. Solnica-Krezel, Curr. Biol. 13, R7 (2003).

[12] Y. Chen and A. F. Schier, Curr. Biol. 12, 2124 (2002).
[13] Y. Chen and A. F. Schier, Nature (London) 411, 607 (2001).

[14] X. Jing, S. Zhou, W. Wang, and Y. Chen, Mech. Develop. 123, 388 (2006).

[15] C. M. Lin, T. X. Jiang, R. E. Baker, P. K. Maini, R. B. Widelitz, and C. M. Chuong, Dev. Biol. 334, 369 (2009).

[16] T. E. Woolley, R. E. Baker, P. K. Maini, J. L. Aragón, and R. A. Barrio, Phys. Rev. E 82, 051929 (2010).

[17] B. Alberts, A. Johnson, J. Lewis, M. Raff, K. Roberts, and P. Walter, Molecular Biology of the Cell, 5th ed. (Garland Science, New York, 2008).

[18] C. N. Tennyson, H. J. Klamut, and R. G. Worton, Nat. Genet. 9, 184 (1995).

[19] J. Lewis, Curr. Biol. 13, 1398 (2003).

[20] M. Kærn, T. R. Elston, W. J. Blake, and J. J. Collins, Nat. Rev. Genet. 6, 451 (2005).

[21] P. S. Swain, M. B. Elowitz, and E. D. Siggia, Proc. Natl. Acad. Sci. USA 99, 12795 (2002).

[22] T. B. Kepler and T. C. Elston, Biophys. J. 81, 3116 (2001). 
[23] J. D. Murray, Mathematical Biology II: Spatial Models and Biomedical Applications, 3rd ed. (Springer-Verlag, Berlin, 2003), Vol. 2.

[24] R. Dillon, P. K. Maini, and H. G. Othmer, J. Math. Biol. 32, 345 (1994).

[25] E. J. Crampin, E. A. Gaffney, and P. K. Maini, Bull. Math. Biol. 61, 1093 (1999).

[26] E. A. Gaffney and N. A. M. Monk, Bull. Math. Biol. 68, 99 (2006).

[27] S. Seirin Lee, E. A. Gaffney, and N. A. M. Monk, Bull. Math. Biol. 72, 2139 (2010).

[28] A. Gierer and H. Meinhardt, Biol. Cybern. 12, 30 (1972).

[29] J. Schnakenberg, J. Theor. Biol. 81, 389 (1979).

[30] J. Higgins, Proc. Natl. Acad. Sci. USA 51, 989 (1964).

[31] E. E. Sel'kov, Eur. J. Biochem. 4, 79 (1968).

[32] E. J. Crampin, E. A. Gaffney, and P. K. Maini, J. Math. Biol. 44, 107 (2002).

[33] A. Sorkin and M. von Zastrow, Nat. Rev. Mol. Cell Biol. 3, 600 (2002).

[34] C. M. Stoscheck and G. Carpenter, J. Cell Biol. 98, 1048 (1984).

[35] C. Le Roy and J. L. Wrana, Dev. Cell. 9, 167 (2005).

[36] J. A. Fischer, S. H. Eun, and B. T. Doolan, Annu. Rev. Cell Dev. Biol. 22, 181 (2006).

[37] T. E. Woolley, R. E. Baker, E. A. Gaffney, and P. K. Maini, Phys. Rev. E 84, 046216 (2011).

[38] M. J. Ward and J. Wei, Stud. Appl. Math. 109, 229 (2002).

[39] D. Iron, J. Wei, and M. Winter, J. Math. Biol. 49, 358 (2004).

[40] R. E. L. DeVille, C. B. Muratov, and E. Vanden-Eijnden, J. Chem. Phys. 124, 231102 (2006).

[41] G. Adomian, Comput. Math. Appl. 29, 1 (1995).
[42] S. Ramsey, D. Orrell, and H. Bolouri, J. Bioinf. Comput. Bio. 3, 415 (2005).

[43] D. T. Gillespie, J. Comput. Phys. 22, 403 (1976).

[44] D. T. Gillespie, J. Phys. Chem. 81, 2340 (1977).

[45] J. Elf and M. Ehrenberg, Genome Res. 13, 2475 (2003).

[46] C. A. Lugo and A. J. McKane, Phys. Rev. E 78, 51911 (2008).

[47] N. G. van Kampen, Stochastic Processes in Physics and Chemistry, 3rd ed. (North-Holland, Amsterdam, 2007).

[48] T. E. Woolley, R. E. Baker, E. A. Gaffney, and P. K. Maini, Phys. Rev. E 84, 021915 (2011).

[49] T. E. Woolley, R. E. Baker, E. A. Gaffney, and P. K. Maini, Phys. Rev. E 84, 041905 (2011).

[50] D. G. Míguez, M. Dolnik, A. P. Muńuzuri, and L. Kramer, Phys. Rev. Lett. 96, 48304 (2006).

[51] T. Marquez-Lago, A. Leier, and K. Burrage, BMC Syst. Biol. 4, 19 (2010).

[52] K. W. Morton and D. F. Mayers, Numerical Solution of Partial Differential Equations: An Introduction (Cambridge University Press, Cambridge, UK, 2005).

[53] T. Tian, K. Burrage, P. M. Burrage, and M. Carletti, J. Comput. Appl. Math. 205, 696 (2007).

[54] S. Seirin Lee and E. A. Gaffney, Bull. Math. Biol. 72, 2161 (2010).

[55] D. Davidson, J. Embryol. Exp. Morph. 74, 245 (1983).

[56] C. Mou, F. Pitel, D. Gourichon, F. Vignoles, A. Tzika, P. Tato, L. Yu, D. W. Burt, B. Bed'hom, M. Tixier-Boichard, K. J. Painter, and D. J. Headon, Plos. Biol. 9, e1001028 (2011).

[57] P. Charvet-Almeida, M. L. G. Araújo, and M. P. Almeida, J. Northw. Atl. Fish. Sci. 35, 165 (2005).

[58] E. M. Ross, M. E. Maguire, T. W. Sturgill, R. L. Biltonen, and A. G. Gilman, J. Biol. Chem. 252, 5761 (1977).

[59] S. Seirin Lee, E. A. Gaffney, and R. E. Baker, Bull. Math. Biol. 73, 2527 (2011). 